\title{
Annex 2: Useful Web sites
}

\author{
Association of Learned and Professional Society Publishers - http://www.alpsp.org \\ CAMiLEON - http://www.si.umich.edu/CAMILEON \\ CEDARS - http://www.leeds.ac.uk/cedars \\ CODATA - http://www.codata.org \\ Council on Library and Information Resources - http://www.clir.org \\ Cross-Industry Working Team - http://www.xiwt.org \\ CrossRef - http://www.crossref.org \\ Deposit of Netherlands Electronic Publications - http://www.kb.nl/dnep \\ Digital Library Federation - http://www.diglib.org \\ Digital Preservation Coalition - www.dpconline.org \\ Digital Preservation Mailing List -www.jiscmail.ac.uk/lists/digital-preservation.html \\ D-Lib Magazine - http://www.dlib.org \\ DOI - http://www.doi.org \\ EDItEUR - http://www.editeur.org \\ ERPANET - http://www.erpanet.org \\ ICSTI - http://www.icsti.org \\ ICSU - http://www.icsu.org \\ ICSU Press - http://associnst.ox.ac.uk/ icsuinfo \\ <indecs>-http://www.indecs.org \\ Internet Archive - http://www.archive.org \\ InterPARES - http://www.interpares.org \\ ISO Archiving Standards - http://ssdoo.gsfc.nasa.gov/nost/isoas \\ Joint Information Systems Committee - http://www.jisc.ac.uk \\ JSTOR - http://www.jstor.org \\ LOCKSS - http://lockss.stanford.edu \\ MINERVA - http://www.amitie.it/minerva \\ NEDLIB - http://www.kb.nl/nedlib \\ OCLC - http://www.oclc.org \\ Open Archives Initiative - http://www.openarchives.org \\ PADI (National Library of Australia) - http://www.nla.gov.au/padi \\ Research Libraries Group - http://www.rlg.org \\ RLG DigiNews - http://www.rlg.org/preserv/diginews \\ U.S. National Archives and Records Administration - http://www.nara.gov \\ UNESCO - http://www.unesco.org \\ World Data Center System - http://www.ngdc.noaa.gov/wdc
}

